\title{
ANALYSIS OF PERIODIC DISCRETE EVENT SYSTEMS IN (MAX,+) ALGEBRA
}

\author{
S. Lahaye, J.L. Boimond, L. Hardouin \\ Laboratoire d'Ingénierie des Systèmes Automatisés, 62 Avenue Notre-Dame du Lac, \\ 49000 Angers, France \\ [lahaye, boimond, hardouin] @istia.univ-angers.fr \\ Keywords: Discrete Event Dynamic Systems, (max,+) Algebra, Periodic Systems
}

\begin{abstract}
Discrete Event Dynamic Systems modeled by $(\max ,+)$ linear equations with periodically varying coefficients are studied. It turns out that spectral properties of the so-called monodromy matrix can be used for the performance evaluation of these systems.
\end{abstract}

\section{INTRODUCTION}

Discrete Event Dynamic Systems (DEDS) subject to synchronization phenomena can be modeled by linear equations in a particular algebraic structure called $(\max ,+)$ algebra. A linear system theory analogous to the conventional theory has been developed for this class of systems which can be, for example, manufacturing systems or communication networks [2]. In particular, linear time invariant systems, whose behaviors are usually represented by Timed Event Graphs with constant timings, have been studied extensively [2], [5], [7]. In a manufacturing system, time invariance corresponds for example to assume that processing times are constant.

Lots of systems arising in practice are time-varying, that is, the values of the output response depend on when the input is applied. Time variation is a result of system parameters changing: in a manufacturing system, processing times of parts may depend on their type. Systems described by state models with varying coefficients in $(\max ,+)$ algebra have been considered in [10]. The output tracking under just-in-time criterion has in particular been extended to such systems.

In this paper, the focus is on linear systems whose state models have periodically varying coefficients. Explicitly, each entry of the matrices 
in the state model satisfy $a(k+K)=a(k), k \in \mathbb{Z}$. We aim at extending to the $(\max ,+)$ context some established concepts and results of the conventional periodic linear system theory [3], [6], [4]. In particular, the spectral properties of the so-called monodromy matrix, i.e., the transition matrix over one period $K$, are used to show that autonomous periodic systems couple in finite time to a periodic regime. This result can for example be applied to the performance evaluation of manufacturing systems in which tasks are scheduled periodically.

The outline of the paper is as follows. In $\S 2$, we recall the elements of $(\max ,+)$ algebra we shall use throughout the paper. In $\S 3,(\max ,+)$ linear time-varying systems are presented. Section 4 is devoted to the analysis of periodic systems. An application to the performance evaluation of a particular class of DEDS is proposed in $\S 5$.

\section{PRELIMINARIES}

We consider the semi-field $(\mathbb{R} \cup\{-\infty\}, \oplus, \otimes)$ in which the law $\oplus$ is $\max$, and $\otimes$ is the usual addition. We denote respectively $\varepsilon=-\infty$ and $e=0$ the neutral elements of $\oplus$ and $\otimes$. The element $\varepsilon$ is absorbing for $\otimes$. The law $\oplus$ is idempotent, i.e., $a \oplus a=a$.

$(\mathbb{R} \cup\{-\infty\}, \oplus, \otimes)$ is an idempotent semi-ring or dioid [2], [5], and is usually referred to as $(\max ,+)$ algebra. We shall denote it by $\mathbb{R}_{\max }$.

In the following, we shall consider vectors and matrices with entries in $\mathbb{R}_{\max }$. The product of a vector $u \in \mathbb{R}_{\max }^{n}$ by a scalar $a \in \mathbb{R}_{\max }$ is defined as $\quad(a \otimes u)_{i}=a \otimes u_{i}=a+u_{i}$.

The sum and product of matrices are defined conventionally, replacing + and $\times$ by $\oplus$ and $\otimes$, respectively. Let $A, B \in \mathbb{R}_{\max }^{n \times n}$,

$(A \oplus B)_{i j}=A_{i j} \oplus B_{i j} \quad(A \otimes B)_{i j}=\bigoplus_{l=1}^{n} A_{i l} \otimes B_{l j}=\max _{1 \leq l \leq n}\left(A_{i l}+B_{l j}\right)$.

The matrix-vector product is defined in a similar way. Most of the time, the symbol ' $\otimes$ ' is omitted as is the case in conventional algebra.

Let us recall basic definitions and results about the $(\max ,+)$ spectral problem (see [2], [7] for exhaustive presentations), that is the existence of (nonzero) eigenvalues $\lambda \in \mathbb{R}_{\max }$ and eigenvectors $v \in \mathbb{R}_{\max }^{n}$ for a given a matrix $M \in \mathbb{R}_{\max }^{n \times n}$, such that $M \otimes v=\lambda \otimes v$.

Definition 1 A matrix $M \in \mathbb{R}_{\max }^{n \times n}$ is irreducible if

$$
\forall i, j \quad \exists l \geq 0 \text { such that }\left(M^{l}\right)_{i j}>\varepsilon .
$$

Theorem 1 An irreducible matrix $M \in \mathbb{R}_{\max }^{n \times n}$ has a unique eigenvalue denoted $\lambda$.

There might be several eigenvectors of an irreducible matrix with the unique corresponding eigenvalue $\lambda$. A linear combination (in $\mathbb{R}_{\max }$ ) of 
eigenvectors is an eigenvector. An eigenvector has all its coordinates different from $\varepsilon$. Finally, let us recall that in $\mathbb{R}_{\max }$ every irreducible matrix is cyclic in the sense of the following theorem.

Theorem 2 Let $M \in \mathbb{R}_{\max }^{n \times n}$ be an irreducible matrix whose eigenvalue is $\lambda$. There exists integers $N$ and $c$ such that

$$
\forall m \geq N, M^{m+c}=\lambda^{c} \otimes M^{m} .
$$

The least value of $c$ is called the cyclicity of $M$.

\section{TIME-VARYING $($ MAX, +$)$ LINEAR SYSTEMS}

We study time-varying $(\max ,+)$ linear systems represented by equations:

$$
\left\{\begin{array}{l}
x(k)=A(k-1) x(k-1) \oplus B(k) u(k) \\
y(k)=C(k) x(k)
\end{array}\right.
$$

in which for $k \in \mathbb{Z}$ :

- $A(k) \in \mathbb{R}_{\max }^{n \times n}, B(k) \in \mathbb{R}_{\max }^{n \times p}$, and $C(k) \in \mathbb{R}_{\max }^{q \times n}$

- $u(k) \in \mathbb{R}_{\max }^{p}\left(\operatorname{resp} . \quad x(k) \in \mathbb{R}_{\max }^{n}, y(k) \in \mathbb{R}_{\max }^{q}\right)$ is called the input (resp. state, output) vector.

The recursive equation (1a) can also be written

$$
x(k)=\Phi\left(k, k_{0}\right) x\left(k_{0}\right) \oplus \bigoplus_{j=k_{0}+1}^{k} \Phi(k, j) B(j) u(j)
$$

in which $\Phi\left(k, k_{0}\right)$ is called transition matrix by analogy with conventional time-varying linear systems theory [8], and is given by

$$
\Phi\left(k, k_{0}\right)= \begin{cases}\text { not defined } & , k_{0}>k \\ \left.I d \quad \text { (identity element of } \mathbb{R}_{\max }^{n \times n}\right) & , k_{0}=k \\ A(k-1) A(k-2) \otimes \cdots \otimes A\left(k_{0}\right) & , k_{0}<k\end{cases}
$$

Remark 1: The transition matrix satisfies the composition property

$$
k \geq l \geq k_{0}, \quad \Phi\left(k, k_{0}\right)=\Phi(k, l) \otimes \Phi\left(l, k_{0}\right) .
$$

In particular, for $k>k_{0}$, we have $\Phi\left(k, k_{0}\right)=A(k-1) \otimes \Phi\left(k-1, k_{0}\right)$, which shows that the transition matrix is solution of the homogeneous state equation (Eq. (1a) with $u(k)=\varepsilon, \forall k$ ).

The input-output relationship is deduced from Eq. (2) with $x\left(k_{0}\right)=$ $u\left(k_{0}\right)=\varepsilon$ for $k_{0}<0$, and is given by $y(k)=\bigoplus_{j \in \mathbb{Z}} h(k, j) u(j)$, with $h(k, j)= \begin{cases}C(k) \Phi(k, j) B(j) & , k \geq j, \\ \varepsilon & , k<j,\end{cases}$ ( $h$ is called the impulse response). 


\section{ANALYSIS OF PERIODIC SYSTEMS}

In this section, we define and study $(\max ,+)$ linear periodic systems by analogy with linear periodic systems over conventional algebra [3], [6], [4]. Using basic properties and $(\max ,+)$ spectral theory, we show that autonomous periodic systems couple in finite time to a periodic regime.

Definition 2 A system represented by Eqs. (1) is said to be periodic of period $K$ (or shortly $K$-periodic) if $K$ is the least integer such that $\forall k \in \mathbb{Z}, A(k+K)=A(k), B(k+K)=B(k), C(k+K)=C(k)$.

Remark 2: The period $K$ of the system is equal to the least common multiplier of the periods of entries $A(k)_{i j}, B(k)_{i k}$ and $C(k)_{l j}, i=1 \ldots n$, $j=1 \ldots n, k=1 \ldots p, l=1 \ldots q, k \in \mathbb{Z}$.

Proposition 1 The transition matrix is K-periodic, i.e.,

$$
k_{0}<k, \Phi\left(k+K, k_{0}+K\right)=\Phi\left(k, k_{0}\right)
$$

if, and only if, $A(k)$ is K-periodic.

Proof: Let us suppose that $A(k)$ is K-periodic. We have $\forall k, k_{0}<k$, $\Phi\left(k+K, k_{0}+K\right)=A(k+K) \otimes \ldots \otimes A\left(k_{0}+1+K\right)=A(k) \otimes \ldots \otimes A\left(k_{0}+1\right)=\Phi\left(k, k_{0}\right)$. Conversely, the K-periodicity of the transition matrix gives for $k_{0}=k-1$

$$
\Phi(k+K, k-1+K)=\Phi(k, k-1),
$$

which, according to the definition of the transition matrix (Eq. (3)), leads to $\forall k, \quad A(k+K)=A(k)$.

The $K$-periodicity of $\Phi$ also writes

$$
k_{0}<k, \forall m \in \mathbb{Z} ; \Phi\left(k+m K, k_{0}+m K\right)=\Phi\left(k, k_{0}\right) .
$$

Setting $k=i+m K$ with $k_{0} \leq i<K, m \in \mathbb{N}$, and using the composition property (4) as well as the periodicity (5) of $\Phi$, we have:

$\Phi\left(i+m K, k_{0}\right)$

$=\Phi\left(i+m K, k_{0}+m K\right) \Phi\left(k_{0}+m K, k_{0}+(m-1) K\right) \otimes \ldots \otimes \Phi\left(k_{0}+K, k_{0}\right)$

$=\Phi\left(i, k_{0}\right) \underbrace{\Phi\left(k_{0}+K, k_{0}\right) \otimes \ldots \otimes \Phi\left(k_{0}+K, k_{0}\right)}_{\text {m times }}$

$=\Phi\left(i, k_{0}\right)\left[\Phi\left(k_{0}+K, k_{0}\right)\right]^{m}$.

Definition 3 The matrix $M_{k_{0}}=\Phi\left(k_{0}+K, k_{0}\right)$ is called the monodromy matrix at $k_{0}$ (as in conventional theory [3]).

For autonomous systems, that is systems for which the input is null $(u(k)=\varepsilon, \forall k \in \mathbb{Z}$ in eq. (1a), the state vector obeys:

$$
\begin{aligned}
x(i+m K) & =\Phi\left(i+m K, k_{0}\right) x\left(k_{0}\right) \\
& =\Phi\left(i, k_{0}\right)\left[\Phi\left(k_{0}+K, k_{0}\right)\right]^{m} x\left(k_{0}\right) \\
& =\Phi\left(i, k_{0}\right) M_{k_{0}}^{m} x\left(k_{0}\right) .
\end{aligned}
$$


In other words, the monodromy matrix describes the evolution of the state over one period. This relation allows showing that an autonomous periodic system couple in finite time to a periodic regime.

Proposition 2 If the monodromy matrix $M_{k_{0}}$ is irreducible with eigenvalue $\lambda$, then there exists two integers $N$ and c such that for $m \geq N$

$$
x(k+(m+c) K)=\lambda^{c} x(k+m K) .
$$

Proof: From equation (6), a direct application of theorem 2 leads to:

$$
\begin{aligned}
x(i+(m+c) K)=\Phi\left(i, k_{0}\right) M_{k_{0}}^{m+c} x\left(k_{0}\right) & =\Phi\left(i, k_{0}\right) \lambda^{c} M_{k_{0}}^{m} x\left(k_{0}\right) \\
& =\lambda^{c} \Phi\left(i, k_{0}\right) M_{k_{0}}^{m} x\left(k_{0}\right) \\
& =\lambda^{c} x(i+m K)
\end{aligned}
$$

in which $\lambda$ (resp. c) is the eigenvalue (resp. the cyclicity) of $M_{k_{0}}$. $\diamond$ In this periodic regime, the length of the pattern is equal to $c \times K$. In the application of section 5 , entries of $x(k)$ shall point out dater variables associated with a DEDS: $x_{i}(k)$ will denote the date of the $k$-th occurrence of event labeled $x_{i}$. The ratio $(c \times \lambda) /(c \times K)=\lambda / K$ (the numerical evaluation of $\lambda^{c}$ in the formula equals $c \times \lambda$ in conventional analysis) shall then be interpreted as the cycle time (inverse of the throughput) of the system; every $c \times K$ occurrences of events are spaced out of $c \times \lambda$ units of times. The following proposition, claiming that the cycle time is independent of $k_{0}$, completes the description of this periodic regime. Remark 3: If $x\left(k_{0}\right)$ is an eigenvector of $M_{k_{0}}$, we have

$$
x\left(k_{0}+K\right)=M_{k_{0}} x\left(k_{0}\right)=\lambda x\left(k_{0}\right) .
$$

From $k_{0}$, the state is then periodic. The pattern is shorter (equal to $K$ ), but the cycle time is still equal to $\lambda / K$.

Proposition 3 The spectrum of $M_{k_{0}}=\Phi\left(k_{0}+K, k_{0}\right)$ is independent of $k_{0}$. Furthermore, if $x\left(k_{0}\right)$ is an eigenvector of $M_{k_{0}}$ with corresponding eigenvalue $\lambda$, then $x(k)=\Phi\left(k, k_{0}\right) x\left(k_{0}\right)$ is an eigenvector of $M_{k}=\Phi(k+$ $K, k)$ with corresponding eigenvalue $\lambda$.

\section{Proof:}

- For any pair $\left(k_{0}, \kappa_{0}\right)$ with $\kappa_{0}+K \geq k_{0} \geq \kappa_{0}$, the monodromy matrices at $k_{0}$ and at $\kappa_{0}$ can respectively be written

- $\Phi\left(k_{0}+K, k_{0}\right)=\Phi\left(k_{0}+K, \kappa_{0}+K\right) \Phi\left(\kappa_{0}+K, k_{0}\right)=\Phi\left(k_{0}, \kappa_{0}\right) \Phi\left(\kappa_{0}+K, k_{0}\right)$

- $\Phi\left(\kappa_{0}+K, \kappa_{0}\right)=\Phi\left(\kappa_{0}+K, k_{0}\right) \Phi\left(k_{0}, \kappa_{0}\right)$

In other words, the monodromy matrices can be expressed in the forms $\Phi\left(k_{0}+K, k_{0}\right)=F G$ and $\Phi\left(\kappa_{0}+K, \kappa_{0}\right)=G F$.

If $\lambda$ is a nonzero eigenvalue of $\Phi\left(k_{0}+K, k_{0}\right)$, i.e., $F G x=\lambda x, x \neq \varepsilon$, then $G F G x=G \lambda x=\lambda G x$, or $G F y=\lambda y$, with $y=G x$.

Since $\lambda \neq \varepsilon$ and $x \neq \varepsilon, y=G x \neq \varepsilon$; so that $\lambda$ is an eigenvalue of $\Phi\left(\kappa_{0}+K, \kappa_{0}\right)=G F$ as well. 
By reversing the role of $\Phi\left(k_{0}+K, k_{0}\right)$ and $\Phi\left(\kappa_{0}+K, \kappa_{0}\right)$ in the above argument, it conversely follows that all the nonzero eigenvalues of $\Phi\left(\kappa_{0}+\right.$ $\left.K, \kappa_{0}\right)$ are eigenvalues of $\Phi\left(k_{0}+K, k_{0}\right)$.

- Let us assume that $x\left(k_{0}\right)$ is an eigenvector of $M_{k_{0}}$ with corresponding eigenvalue $\lambda$. We have

$$
\begin{aligned}
M_{k} x(k)=\Phi(k+K, k) x(k) & =\Phi(k+K, k) \Phi\left(k, k_{0}\right) x\left(k_{0}\right) \\
& =\Phi\left(k+K, k_{0}\right) x\left(k_{0}\right) \\
& =\Phi\left(k+K, k_{0}+K\right) \Phi\left(k_{0}+K, k_{0}\right) x\left(k_{0}\right) \\
& =\Phi\left(k, k_{0}\right) \lambda x\left(k_{0}\right) \\
& =\lambda x(k)
\end{aligned}
$$

which shows that $x(k)$ is an eigenvector of $M_{k}$ with corresponding eigenvalue $\lambda$.

\section{APPLICATION TO THE PERFORMANCE EVALUATION OF DEDS}

In this section, we apply the preceding results to DEDS. More precisely, we define a class of Timed Petri Nets suitable to model timevarying $(\max ,+)$ linear systems (introduced in [10]). Essentially, those are Timed Event Graphs (TEGs, Petri net for which each place has only one input arc and one output arc) whose holding times associated with places are variable. We give their representation in $(\max ,+)$ algebra. When sequences of holding times are periodic, the representation is a state model with periodically varying coefficients.

\subsection{FIFO TEGS}

We denote by $\mathcal{P}$ (respectively, $\mathcal{Q}$ ) the finite set of places (respectively, transitions) of a TEG, and $M_{p} \in \mathbb{N}$ the number of tokens being initially in place $p \in \mathcal{P} ; p^{\bullet}$ (respectively, ${ }^{\bullet} p$ ) refers to the output transition (respectively, input transition) of $p$. We define similarly the sets $q^{\bullet},{ }^{\bullet} q$ as the set of output places, and the set of input places, of transition $q \in \mathcal{Q}$.

We call holding time the minimum amount of time tokens have to stay in a place (without loss of modeling power, the firing of transitions is supposed to be instantaneous): the $k$-th token in place $p$ incurs the holding time denoted $\tau_{p}(k)$.

Definition 4 We define the earliest First-In-First-Out (FIFO) functioning rule of a TEG as follows.

1 A transition $q$ fires as soon as each place upstream $q$ contains at least one available token. 
2 We denote $q(n)$ the date of $n$-th firing of transition $q$. This firing consumes one token in each upstream place and produces one token in each downstream place. A token added in place $p \in q^{\bullet}$ at time $q(n)$ is indexed $k$ with $k=n+M_{p}$ and becomes available for transition $p^{\bullet}$ from instant $\max _{1 \leq i \leq n}\left\{q(i)+\tau_{p}\left(i+M_{p}\right)\right\}$.

A TEG functioning under this rule is called a FIFO TEG.

The only originality in this definition concerns the token's availability. Indeed, the token indexed $k$ in place $p$ is usually said to be available as soon as its holding time $\tau_{p}(k)$ is over [1].

The reason why we have defined a new functioning rule is that TEGs can be modeled by linear equations in $(\max ,+)$ algebra only if tokens do not overtake one another when traversing places [1], [2, ch. 2]. Previous studies have consequently considered conditions on sequences of holding times (constant or non-decreasing holding times for example)and/or structural conditions (preventing several tokens to be simultaneously present in a place with variable holding time). The above functioning rule ensures without structural conditions that places operate as FIFO channels for any sequences of holding times, and will notably enable to easily model mixed-model assembly lines (which are intrinsically overtake free) on which several parts can be simultaneously handled at a same "station" or machine. Let us consider for example the automobile production line partly shown in figure 1.(a) where cars are handled by a linear accumulative conveyor crossing successive working areas separated by buffer zones. In each working area, a fixed number of cars

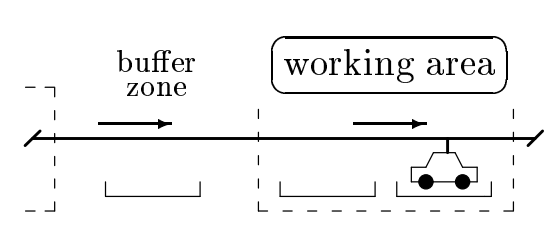

(a)

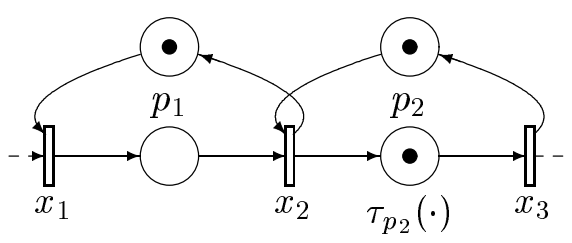

(b)

Figure 1 (a) A portion of an automobile production line, (b) a FIFO TEG.

can be processed simultaneously (two in the represented working area) and buffer zones have limited capacities (only one car can be stocked in the represented buffer zone). Cars cannot overtake one another on the conveyor; buffer zones and working areas work as FIFO channels. The evolution of cars in the considered portion of the line can be modeled by the FIFO TEG of figure 1.(b). A token in place $p_{1}$ (resp. $p_{2}$ ) represents 
a car stocked in the buffer zone (resp. being processed in the working area). Processing times in the working area are equal to $\tau_{p_{2}}(\cdot)$, and for sake of simplicity, travelling time in the buffer zone as well as setup times are assumed to be null. Finally, let us note that this system would not be modeled by a TEG if the classical functioning rule was used.

\subsection{REPRESENTATION OF FIFO TEGS}

With each transition $q \in Q$ we associate a dater variable also denoted $q: q(k)$ denotes the date of the $k$-th firing of transition $q$. The sequences of holding times $\tau_{p}(k), p \in \mathcal{P}, k \in \mathbb{Z}$ are assumed to be given nonnegative and finite integers.

Assertion 1 The dater variables of a FIFO TEG satisfy the following equation:

or equivalently,

$$
q(k)=\bigoplus_{\left\{p \in \cdot \bullet_{\left.q \mid q^{\prime}=\bullet p\right\}}\right.} \bigoplus_{i \leq k}\left[\tau_{p}(i) \otimes q^{\prime}\left(i-M_{p}\right)\right], k \in \mathbb{Z},
$$

$$
q(k)=q(k-1) \oplus \bigoplus_{\left\{p \in \bullet_{\left.q \mid q^{\prime}=\bullet p\right\}}\right.}\left[\tau_{p}(k) \otimes q^{\prime}\left(k-M_{p}\right)\right], k \in \mathbb{Z} .
$$

The methodology to obtain a state representation for FIFO TEGs in $(\max ,+)$ algebra is the same one as for TEGs functioning with the classical rule [2, chap. 2]. One can partition the set of transitions $\mathcal{Q}=\mathcal{U} \cup \mathcal{X} \cup \mathcal{Y}$ where $\mathcal{U}$ is the set of transitions with no predecessors (input transitions), $\mathcal{Y}$ is the set of transitions with no successors (output transitions), and $\mathcal{X}=\mathcal{Q} \backslash(\mathcal{U} \cup \mathcal{Y})$ (state transitions). We denote by $u$ (respectively $x, y$ ) the vector of input (respectively, state, output) daters $q, q \in \mathcal{U}$ (respectively, $\mathcal{X}, \mathcal{Y}$ ). One can obtain after several combinatorial manipulations the standard state model given by Eqs. (1) (see [10]).

Example 1: We consider the FIFO TEG of figure 1.(b) (non-dotted part of the graph) which may partly represent a working area of an automobile production line, as described in section 5.1. This graph has exclusively state transitions, its dynamic behavior can be represented by the following equation:

$$
x(k)=\left(\begin{array}{l}
x_{1}(k) \\
x_{2}(k) \\
x_{3}(k)
\end{array}\right)=\left(\begin{array}{lll}
\varepsilon & \varepsilon & \varepsilon \\
e & \varepsilon & \varepsilon \\
\varepsilon & \varepsilon & \varepsilon
\end{array}\right) \otimes\left(\begin{array}{l}
x_{1}(k) \\
x_{2}(k) \\
x_{3}(k)
\end{array}\right) \oplus\left(\begin{array}{ccc}
e & e & \varepsilon \\
\varepsilon & e & e \\
\varepsilon & \tau_{p_{2}}(k) & e
\end{array}\right) \otimes\left(\begin{array}{l}
x_{1}(k-1) \\
x_{2}(k-1) \\
x_{3}(k-1)
\end{array}\right) .
$$

This equ. can be written in an explicit form (see [2, th. 2.66, p. 79]):

$$
x(k)=\left(\begin{array}{l}
x_{1}(k) \\
x_{2}(k) \\
x_{3}(k)
\end{array}\right)=\left(\begin{array}{ccc}
e & e & \varepsilon \\
e & e & e \\
\varepsilon & \tau_{p_{2}}(k) & e
\end{array}\right) \otimes\left(\begin{array}{l}
x_{1}(k-1) \\
x_{2}(k-1) \\
x_{3}(k-1)
\end{array}\right) .
$$


A FIFO TEG represented by Eqs. (1) will be seen as a periodic system if, and only if, all the entries of matrices $A(k), B(k)$ and $C(k)$ (corresponding to the holding times associated with places of the graph) are periodic. The periodicity of the system, denoted $K$, is then equal to the lowest common multiplier of the periodicities of the sequences of holding times. In the manufacturing system described above, sequences of holding times represent the processing times of cars crossing the working area. Such a system is then periodic if for example the successive types of cars released on the production line are ordered in a periodic manner. In such a periodic setting, the reasoning of section 4 can be used to show that daters associated with transitions of the FIFO TEG reach a periodic regime, and to assess the cycle time of these daters which correspond to the average time between two successive firings.

Example 2: Let us consider again the portion of the automobile production line modeled in example 1.

We assume that three types of cars, denoted $R_{1}, R_{2}$ and $R_{3}$, are handled in this line. The processing time for $R_{1}$ (resp. $R_{2}, R_{3}$ ) in the considered working area is equal to 3 (resp. 2,1) units of times. The scheduling is supposed to be a cyclic permutation of the different types of cars. More precisely, the successive types of cars released in the line are : $R_{1}, R_{2}$, $R_{3}, R_{1}, R_{2}, R_{3}, \ldots$ We then have $\forall j \in \mathbb{Z}$,

$\tau_{p_{2}}\left(k_{0}+3 j\right)=3, \quad \tau_{p_{2}}\left(k_{0}+3 j+1\right)=2, \quad \tau_{p_{2}}\left(k_{0}+3 j+2\right)=1$.

The system is 3 -periodic since $A(k+3)=A(k), \forall k \in \mathbb{Z}$. The monodromy matrix at $k_{0}$ is equal to

$$
M_{k_{0}}=\Phi\left(k_{0}+3, k_{0}\right)=\left(\begin{array}{ccc}
0 & 3 & 0 \\
2 & 3 & 2 \\
2 & 4 & 2
\end{array}\right) .
$$

$M_{k_{0}}$ is irreducible (see definition 1). Its unique eigenvalue $\lambda$ is equal to 3 and its cyclicity $c$ is equal to 1 . The state of the systems reaches a periodic regime in finite time. The length of the pattern is equal to $c \times K=1 \times 3=3$ and the cycle time of the system is equal to $\lambda / K=3 / 3=1$.

Remark 4: Repetitive manufacturing systems have previously been studied in [9]. Nevertheless, the approach presented in this reference does not allow considering systems where several parts can be handled simultaneously on a same machine as in the considered example.

\section{CONCLUSION}

We have tackled the study of $(\max ,+)$ linear systems with periodically varying parameters. We have given basic properties, and in particular we have shown that such autonomous systems reach a periodic regime 
in finite time. This result can be used for the performance evaluation of particular DEDS.

We think that further results on conventional periodic systems could be adapted to the considered DEDS. In particular, for the analysis of discrete time periodic systems, it is often useful to resort to a time-invariant reformulation [6], [4]. In a similar way, $(\max ,+)$ linear periodic systems admit a time-invariant reformulation which could extend the study of these systems.

\section{References}

[1] F. Baccelli. Ergodic theory of stochastic Petri networks. Annals of Probability, 20(1):375-396, 1992.

[2] F. Baccelli, G. Cohen, G.J. Olsder, and J.P. Quadrat. Synchronization and Linearity. Wiley, 1992.

[3] S. Bittanti. Deterministic and stochastic linear periodic systems. In Springer, editor, Time series and linear systems, pages 141-182. 1996.

[4] P. Bolzern, P. Colaneri, and R. Scatolini. Zeros of discrete-time linear periodic systems. IEEE Transactions on Automatic Control, 31:1057-1058, 1986.

[5] G. Cohen, P. Moller, J.P. Quadrat, and M. Viot. Algebraic tools for the performance evaluation of discrete event systems. IEEE Proceedings: Special issue on Discrete Event Systems, 77(1), Jan. 1989.

[6] P. Colaneri, R. Scatolini, and N. Schiavoni. Stabilization, regulation, and optimization of multirate sampled-data systems. In C.T. Leondes, editor, Control and Dynamic Systems, volume 71, pages 95-130. Academic Press, 1995.

[7] S. Gaubert. Théorie des systèmes linéaires dans les dioïdes. Thèse, Ecole des Mines de Paris, July 1992.

[8] E. W. Kamen. Fundamentals of linear time-varying systems. In W. S. Levine, editor, The Control Handbook. IEEE Press and CRC Press, 1996.

[9] S. Laftit, J.M. Proth, and X.L. Xie. Optimization of invariant criteria for event graphs. IEEE Transactions on Automatic Control, 37:547-555, 1992.

[10] S. Lahaye, J.L. Boimond, and L. Hardouin. Just in time control of time-varying discrete event dynamic systems in $(\max ,+)$ algebra. Internal Report R-99-01, LISA, 1999. 